\title{
Endoscopía digestiva alta en pacientes pediátricos
}

\author{
Wilson Daza C.'; Eduardo Chávez C. ${ }^{2}$; \\ Sergio Ceresa O.,3 Bárbara Pizarro M. ${ }^{2}$
}

\begin{abstract}
Resumen
Se descriten las indicociones médicas y 'os hal'azgos de las endoscopías digestivas alras er los pociertes aterididos en un policlinico de gasliperterocgía pediálica de un hespitcl general de Santiago Metropolitano entre juio de 1992 y diembre de 1995. Se hicieron 926 endoscopias alias, 385 en niños y 54 len niñas. la edad de los pacientes oscilabo entre 1 res y 15 años $\{6,7 \pm 4,5$ oños\}. Lc maycría de los procedimientos se indicó por dolor abcominal ecurente $(20,8 \%$, con el cbjetivo de descartar alecciones orgánicas: en lactanles por refluja gasiro. esclagico $\{35,3 \%\}$; en preescolares la causo más frecuente fue hiperlensión porlal $\{46,7 \%$; en escolares se incicć prinejpalmente por dolor abdomiral recurrente $[25 \%$ y en cdoescentes por hemorrogia digestiva alta $(37,5 \%$. En un cuarlo de los casos los resuitados de la endoscopic lueron normales; a esofagitis fue el a allazgo endoscócico más

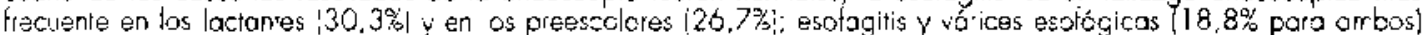
en escolares y as ulceros duodencles en los adolescentes $(20,2 \%)$, en concordoncia con las signos elínicos que motivaron la ind cación to endoscapio degestiva alta es un mélodo paco complejo y útil en el diacnéstico diferencial de os enfermedades gostrointestinales de os niños.
\end{abstract}

(Palabras clavo: endoscopia, sistema digesivo, gastrointestira, dolor abdominal, reflujo gastroesolágico, várices esołógiccs.)

\section{Upper gastrointestinal endoscopy}

This sudy was done to describe indications and of upper gastrointestinal ract endoscopy in children at a pediatric gesscenre ology outpatiant clinic of a vniversity associated gene'al hospital at Sontiago, Chile. Along a 30 month pe'ıod extending from uly 1992 thecughoul decemoer 1995, 923 upper gastrcintestinal tract endoscopies were done 10385 male and 541 ferrale palien's aged $0.7 \pm 4.5$ years \{range i month. 15 yeass). Abdominal pain was the most frequent indication cs a whole $20.8 \%$ for upper gastointestirial iract endoscopy. The mast tequent indication for the procedure in children cged 11024 monl'ns was gostrososophageal reflux $\{35.3 \%$, while in 2106 year olds it was porlal hypertension $\{46.7 \%$, being ajain abdominal poin in school oge patients $\{25 \%\}$ and gastrointestinal bleeding 137.5\% in adolescent subjocts. Esophagilis was the most common endoscopic linding in bot: 1 ic 24 month old 130.3\% in ants and toddlers and 2 to $b$ yea ald $(20.7 \%)$ children. In school age patien's, both esophagitis and esophagheal varices were the most frequent endoscopic findings (18.8\%. while duodenol ulcer was the main problem omong older child en $29.2 \%$ ! L'pper digestive trac: eridoscopy in children is nol a difficult procedure and constilutes a useful tool in 1re diferertal diagnosis $n$ some of their gastroirtesinal ciseoses.

'Key words: endascopy, digeslive system, gastrointesinal: abdominal poin, gasirzesophageal reflux, esophageal vari ces.l

Durante las dos últimas décadas, la endoscopia digestiva alta se ha desartollado como uno de los métodos más usados en gastroenterología pediátrica, ha permitido entender mejor las en-

I. Becado. Unidad de Gastroenterologia Infantil, Hospital Clínico San Borja-Arriarón.

2. Unidad de Gastroenterología Infantil. Hospital Clínico San Borja-Arriarán.

3. Unidad de Gastroenterología, Clinica Las Condes. fermedades del sistema gastrointestinal en los niños, hacer mâs sencillo el diagnóstico diferencial e incluso realizat intervenciones terapéutica en algunas enfermedades'. Es, ademâs, un examen seguro y simple $e^{2}$.

En este trabajo se describen los motivos de consulta por los cuales se ha indicado el procedimiento en niños atendidos en un policlónico de gastroenterología de un servicio de pediatría en un hospital general y su rendimiento en térmi- 
nos de los hallazgos endoscópicos correspondientes.

\section{Pacientes y Métodos}

Se revisaron las esófago-gastro-duodenoscopías realizadas desde julio de 1992 hasta djciembre de 1995, incluyendo 926 procedimientos: 385 en niños y $\$ 41$ en niñas, derivados a la unidad de gastroenterologia infantil del Hospital Clínico San Borja-Arriarán. Los pacientes fueron sedados con midazolam intravenoso $(0,2 \mathrm{mg}$ - $\mathrm{kg}$ peso hasta un máximo de $2,5 \mathrm{mg}$ como dosis total). Además, a todos los pacientes se les roció la faringe posterior con lidocaína en pulverizador. La endoscopía fue realizada por los gastroenterólogos de la unidad mediante un fibroscopio Olympus XP-20 con un OES Lecture Scope LS-10. El procedimiento fue registrado en un protocolo estándar, en el cual se describen las observaciones hechas en esófago, estómago y duodeno. El análisis de la información así registrada se efectuó con un programa estadístico Systat; los datos se expresaron en porcentajes y algunas medidas de tendencia central (promedio) y de dispersión (desviación estándar).

\section{Resultados}

La edad de los pacientes atendidos oscilaba entre 1 mes y 15 años, promedio $6,7 \pm 4,5$ años. En $23,9 \%$ de los procedimientos el paciente era un lactante (niño menor de 2 años de edad), en $20,8 \%$ preescolar ( 2 a 5 años), en 22,1 \% escolar (mís de 5 a 12 años) y en $33,2 \%$ adolescente (más de 12 y hasta 15 años).

Del total de 926 endoscopías digestivas altas, la mayoría fue indicada por dolor abdominal recurrente $(20,8 \%)$, con el propósito de descartar enfermedades orgánicas, seguida en segundo lugar por hemorragia digestiva alta y luego por signos o síntomas de hipertensión portal y con el propósito de pesquisar várices esofágicas (tabla 1).

En $25 \%$ de las endoscopías los resultados fueron normales (tabla 2). La tabla 3 muestra que la mayoría de los procedimientos en los lactantes fue indicada por reflujo gastroesofágico $(35,3 \%)$, en los preescolares por hiper-

\section{Tabla 1}

Indicaciones clínicas en 926 endoscopías digestivas altas en niños

\begin{tabular}{lll} 
Clínica & & \\
\hline Refluja gastro-esofágico & 118 & 12.7 \\
Hipertensión portal & 167 & 18,0 \\
Hemorragia digestiva alta & 168 & 18,1 \\
Doior abdominal recurtente & 192 & 20.8 \\
Síndrone de malabsorción & 194 & 16.7 \\
Otros & 127 & 13.7 \\
\hline Toral & 926 & 100,0 \\
\end{tabular}

Tabla 2

Hallazgos en 926 endoscopias digestivas altas en niños

\begin{tabular}{|c|c|c|}
\hline Hallazgos & $\mathbf{n}$ & $\%$ \\
\hline Normal & $23 !$ & 25,0 \\
\hline Esofagitis & 182 & 19,7 \\
\hline Varices esofágicas & 115 & 12,4 \\
\hline Gastritis & 65 & 7,0 \\
\hline Duodenitis & 115 & 12,4 \\
\hline Uleera duodenat & 90 & 9,7 \\
\hline Otros & $\mathrm{t} 28$ & $1.3,8$ \\
\hline Total & 926 & 100.0 \\
\hline
\end{tabular}

tensión portal (46.7\%), en los escolares por dolor abdominal recumente (25\%) y en los adolescentes por hemorragia digestiva alta $(37,5 \%)$. Los diagnósticos endoscópicos más frecuentes (tabla 4) fueron esofagitis en los lactantes $(30,3 \%)$ y en los preescolares $(26,7 \%)$; esofagitis y várices esofágicas $(18,8 \%$ cada uno) en escolares y úlcera duodenal en los adolescentes $(29,2 \%)$. 
Tabla 3

Indicaciones de endoscopias altas según edad en 926 procedimientos en njños

\begin{tabular}{lccccccrr}
\hline Edad & \multicolumn{2}{c}{ Lactante } & \multicolumn{2}{c}{ Preescolar } & \multicolumn{2}{c}{ Escolar } & \multicolumn{2}{c}{ Adolescente } \\
Indicaciones & $\mathbf{n}$ & $\%$ & $\mathbf{n}$ & $\%$ & $\mathbf{n}$ & $\%$ & $\mathbf{n}$ & $\boldsymbol{\%}$ \\
\hline RGE & 78 & $\mathbf{3 5}, 3$ & - & - & 26 & 12,5 & 26 & $\mathbf{8 . 3}$ \\
HTP & 13 & 5,9 & 90 & 46,7 & 38 & 18,7 & - & - \\
HVDA & 27 & 12,2 & 13 & 6,7 & 26 & 12,5 & 115 & 37,5 \\
DAR & 13 & 5,9 & 26 & 13,3 & 51 & 25,0 & 102 & 33,4 \\
SMA & 64 & 28,9 & 38 & 20,0 & 13 & 6,3 & 38 & 12,5 \\
Otro & 26 & 11,8 & 26 & 13,3 & 51 & 25,0 & $\mathbf{2 6}$ & $\mathbf{8 , 3}$ \\
\hline Total & 221 & 100,0 & 193 & 100,0 & 205 & 100,0 & 307 & 100,0 \\
\hline
\end{tabular}

RGE: reflujo gastroesofágico; HTP: hipertensión poral, HVDA: hemoragia vfa digestiva alta; DAR: dolor abdominal recutrente; SMA: sindrome de malabsorcion.

\section{Tabla 4}

Hallazgos en 926 endoscopías gastrointestinales altas según edad

\begin{tabular}{|c|c|c|c|c|c|c|c|c|}
\hline \multirow{2}{*}{$\begin{array}{l}\text { Edad } \\
\text { Hallazgos }\end{array}$} & \multicolumn{2}{|c|}{ Lactante } & \multicolumn{2}{|c|}{ Preescolar } & \multicolumn{2}{|c|}{ Escolar } & \multicolumn{2}{|c|}{ Adolescente } \\
\hline & $\mathbf{n}$ & $\%$ & $\mathbf{n}$ & \% & $\mathbf{n}$ & $\%$ & $\mathbf{n}$ & $\%$ \\
\hline Nonnal & 89 & 40,2 & 64 & $3,3,2$ & 51 & 25,0 & 51 & 16,7 \\
\hline Esofagitis & 67 & 30,3 & 52 & 26,9 & 38 & 18.8 & 39 & 12,5 \\
\hline Várices esofágicas & 26 & 11,8 & 38 & 19,8 & 38 & 18,8 & - & - \\
\hline Gastritis & - & - & 13 & 6.7 & 13 & 6,2 & 38 & 12.5 \\
\hline Duodenitis & 26 & 11.8 & 13 & 6.7 & - & - & 64 & 20,8 \\
\hline Ulcera duodenal & - & - & - & - & 13 & 6,2 & 90 & 29,2 \\
\hline Otros & 13 & 5,9 & 13 & 6.7 & SI & 25,0 & 25 & 8,3 \\
\hline Total & 221 & 100.0 & 193 & 100.0 & 204 & 100,0 & 307 & 100,0 \\
\hline
\end{tabular}

\section{Comentario}

Antes de la introducción de la endoscopía digestiva en los niños, la mitad de los casos con hematemesis, melena o dolor abdominal quedaban sin diagnóstico etiológico ${ }^{3}$.

Entre las diferentes indicaciones para la endoscopía digestiva en niños, algunas son de carácter diagnostico y otras terapéuticas (por ejemplo: escleroterapia de várices esofágicas, gastrostomía percutánea, extracción de cuerpo extraño).

A pesar que en $90 \%$ de los casos de dolor abdominal recurrente en niños no se identifica una causa orgánica ${ }^{4}$, este síntoma fue el motivo de consulta más frecuente por el cual se solicitó la endoscopja a nuestros pacientes y a los de otras series ${ }^{5,6}$. La importancia del diagnóstico oportuno de várices esofágicas para su tratamiento precoz $z^{7-9}$ es muy probablemente el motivo de que la mayoría de las endoscopías digestivas altas en preescolares y escolares se indique a causa de hipertension portal. La concordancia entre hemorragia digestiva alta $\rightarrow$ como indicación principal de endoscopía- y la úlcera duodenal -como hallazgo endoscópico predominante- en los adolescentes muestra que, aunque en pediatría no es tan frecuente la enfermedad ácido-péptica como en adultos, es importante tenerla presente, especialmente en dicha fase del desarrollo, debido a que su incidencia está aumentando ${ }^{10}$. 


\section{Referencias}

I. Cremer M, Peeters SP, Emomis P. Rodesch P Cadratiel $S$ : Fiberendoscopy of the gastrointestinal tract in children. Experience with newly designed fiberscope. Endoscopy 1974; 6: 186-189.

2. Amest $M E$, Christie DL: Upper gastrointestinal fiberoptic endoscopy in pediatric patients. Gasiroenterology 1977: 72: 1244-1248.

3. Ament ME. Vorgas $J$ : Fiberoptic upper intestinal endoscopy. In: Pedjatric Gastrointestinal Disease. Walker-Smitt WA. Durie PR, Hamilton JR. WalkerSmith JA. Watkins J. Philadelphia, Toronto, 2nd ed, Ed. BC Decker Inc 1991: 1247-1256

4. Apley $J$ : The child with abdominal pains. $20 d$ ed. Oxford: Blackwell Scientific Publications 1975.

5. Kohli $Y$, Fisse Y. Kodana T, el al: Upper gastrointestinal endoscopy in pediatric patients. Gastroenterol Endosc 1981; 23: 1294-1301.
6. Prolla JC, Diehi AS. Benvenufi GA. Loguercio $S V$. Magalhaes DS. Sllveita TR: Upper gastrointestinal fiberoptic endoscopy in pediatric patients. Gastrointest Endose 1983; 29: 279-281.

7. Pizary B. Llorens P, Rios $G$, Cháyez E. Ceresa S: Escleroterapia (ET) de várices esofágicas en niños. Rev Chil Pediatr 1993; 64 (resúmenes de congreso): $\mathrm{N}^{\circ} \mathrm{GT}-08$.

8. Carvajal S, González R, Rollin A: Escleroterapia en varices esofágicas sangrantes: efectividad, complicaciones y mortaljdad. Gastroenterología Latinoamericanin 1993; 4: resumen TL 17.

9. Stringer MD. Homard ER: Long lerm outcome after injection scletotherapy for oesophageal varices in children with extrahepatic portal hypertension. Gut 1994: 35: 257-2.59.

10. Druemm B. Rhotads JM. Stinger D. Sherman P. Ellis L. Duric P: Peptic ulcer disease in thildren: eljology. clinical findings and clinical course. Pediatrics 1988; 82: $410-414$.

\section{AVISO A LOS AUTORES}

Con el objeto de acelerar la edición de los trabajos se solicita encarecidamente a los autores que disponen de computador con procesadores de texto IBM compatibles, enviar -en lo posible junto con las dos copias impresas de su material-, otra en disco flexible (tamaño 3,5" o 5,25", de doble o alta densidad) del mismo texto. El disco será devuelto de inmediato por correo certificado o por mano al autor corresponsal. 